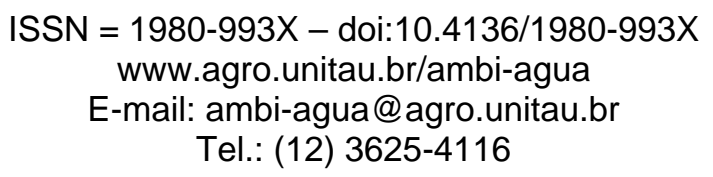

\title{
Efeitos da irrigação e do nitrogênio na produtividade do trigo
}

(doi:10.4136/ambi-agua.5)

\section{Bernardo Friedrich Theodor Rudorff'; Mauricio Alves Moreira' ${ }^{\mathbf{1}}$; Marcelo dos Santos Targa ${ }^{2}$; José Guilherme de Freitas ${ }^{3}$}

\author{
${ }^{1}$ Instituto Nacional de Pesquisas Espaciais (INPE), Divisão de Sensoriamento Remoto \\ Av. dos Astronautas, 1.758, CEP 12.227-010, São José dos Campos, SP \\ E-mail: \{bernardo, mauricio\}@ltid.inpe.br \\ 2Universidade de Taubaté (UNITAU), Departamento de Ciências Agrárias \\ Estr. Dr. José Luiz Cembranelli, 5.000, CEP 12081-010, Taubaté, SP \\ E-mail: mtarga@agro.unitau.br \\ 3Instituto Agronômico de Campinas (IAC) \\ Av. Barão de Itapura, 1.481, Caixa Postal 28, 13020-902, Campinas, SP \\ E-mail: jfreitas@iac.sp.gov.br
}

\section{RESUMO}

Dois experimentos agronômicos com a cultura do trigo foram conduzidos durante o período de maio a outubro de 1999 e 2000 na área experimental da Universidade de Taubaté, Taubaté, SP. O objetivo do trabalho foi avaliar o efeito de diferentes níveis de irrigação e doses de adubação nitrogenada sobre a produtividade de grãos de dois cultivares de trigo. $\mathrm{O}$ trabalho pretende também apresentar a viabilidade dessa atividade agrícola durante a entressafra na região do Vale do Paraíba no Estado de São Paulo. O delineamento experimental permitiu simular a campo diferentes condições de crescimento da cultura do trigo em termos da interação dos fatores irrigação, cultivar e adubação nitrogenada. Os resultados mostraram que não houve interação significativa entre estes três fatores para as características agronômicas analisadas. A interação da irrigação com a adubação nitrogenada foi significativa apenas em 1999 para produtividade de grãos e fitomassa. A fertilidade do solo foi melhorada em 2000 com um efeito positivo sobre a produtividade de grãos. A irrigação teve um impacto significativo sobre a produtividade de grãos, indicando que a cultura do trigo precisa ser suprida com água para uma produção rentável na região do Vale do Paraíba. O balanço hídrico é recomendável para uma irrigação eficiente.

Palavras-chave: experimento agronômico; Vale do Paraíba; agricultura; cultivares de trigo.

\section{Irrigation and nitrogen effects on wheat grain yield}

\begin{abstract}
Two agronomic experiments with wheat crop were conducted during the period of May through October of 1999 and 2000 at the experimental site of the University of Taubaté, Taubaté, São Paulo State, Brazil. The objective of this work was to evaluate the effect of different levels of irrigation and nitrogen on grain yield of two wheat cultivars. The work also intended to present the feasibility of this agriculture activity during the winter crop season in the Paraíba Valley region of São Paulo State. The experimental design allowed simulating different wheat crop growth conditions in the field in terms of the interactions among irrigation, cultivar and nitrogen factors. Results showed no significant interaction among the three factors for the analyzed agronomic characteristics. Interaction of irrigation and nitrogen, for grain yield and biomass, was observed only in 1999. Soil fertility was improved in 2000 with a positive effect on grain yield. Irrigation had a significant impact on grain yield
\end{abstract}


indicating that wheat crop needs water supply for a profitable production in the Paraíba Valley region. Water balance is recommended for efficient irrigation.

Keywords: agronomic experiments; Paraíba Valley; agriculture; wheat cultivars.

\section{INTRODUÇÃO}

No Vale do Paraíba, a cultura do trigo pode ser uma opção de cultivo para o período março-setembro em sucessão às culturas tradicionalmente cultivadas no verão. Conforme Felicio et al. (2001), o cultivo de trigo no Estado de São Paulo, até o início da década de 80, instalava-se no Vale do Paranapanema e na região sul do estado, onde o trigo era semeado em sucessão à soja e cultivado em condições de sequeiro. Contudo, em regiões do Brasil como o norte e oeste do Paraná, sudeste de São Paulo e sul do Mato Grosso do Sul, a deficiência hídrica é mais freqüente, e pode ocasionar perdas totais em determinados anos (Embrapa, 1981). Por outro lado, nessas regiões, bem como no Brasil Central, onde as precipitações são insuficientes para o desenvolvimento normal da cultura, alguns experimentos mostram resposta positiva da cultura do trigo à irrigação (Ferreira et al., 1973; Silva; Leite, 1975; Silva, 1978; Frizzone et al., 1985). No Estado de São Paulo, especificamente a partir de 1982, com o estabelecimento da irrigação, a deficiência hídrica deixou de ser uma limitação para o cultivo do trigo durante o inverno paulista.

Ao analisar os valores médios de uma série temporal de dados entre 1982 e 1992 de um posto meteorológico situado no município de Taubaté, SP, Fisch (1995) observou que a baixa demanda evapotranspirativa das plantas durante a estação seca no inverno pode ser suprida pela água retida no solo nos meses de maiores precipitações. Contudo, os valores de precipitação dessa série temporal de dados apresentam uma significativa variação interanual, em especial nos meses mais secos, levando a concluir que o déficit hídrico ocorre com freqüência apesar da baixa demanda evapotranspirativa.

Níveis adequados de irrigação e adubação nitrogenada que proporcionem um sinergismo desses dois fatores sobre a produção do trigo ainda necessitam de estudos mais refinados a fim de fornecer ao agricultor práticas de manejo adequadas para otimizar o uso dos recursos e obter uma produção rentável (Frizzone et al., 1996). Além disso, devem ser observados cuidados na aplicação do adubo nitrogenado e da irrigação a fim de maximizar o uso desses recursos (Boaretto et al., 2000). Em experimentos a campo e em casa de vegetação foi verificado que o estresse hídrico e a adubação nitrogenada tiveram uma interação negativa sobre a produção de trigo (Camargo, 1976; Parameswaran et al., 1984), pois o principal mecanismo de absorção de nitrogênio é o fluxo de massa, ou seja, quanto mais água for absorvida pelas plantas de trigo, mais nitrogênio da solução do solo será absorvido (Epstein, 1975). Rodrigues et al. (1998), estudando o efeito da deficiência hídrica na produtividade de trigo, concluíram que o número de grãos por espiga é o componente da produtividade mais afetado pela deficiência hídrica.

O presente estudo tem por objetivo analisar o efeito interativo de diferentes níveis de irrigação e doses de nitrogênio sobre a produtividade de grãos de dois cultivares de trigo. $\mathrm{O}$ estudo também tem a finalidade de verificar se o cultivo do trigo é viável na entressafra na região do Vale do Paraíba para permitir uma atividade agrícola rentável por meio do uso otimizado dos recursos naturais e assim minimizar o impacto ambiental. 


\section{MATERIAL E MÉTODOS}

Os experimentos agronômicos a campo com a cultura do trigo foram conduzidos durante o período de maio a outubro de 1999 e 2000 na área experimental do Departamento de Ciências Agrárias da Universidade de Taubaté (UNITAU), em Taubaté, São Paulo, com as seguintes coordenadas geográficas: latitude de $22^{0} 54^{\prime} \mathrm{S}$, longitude de $45^{0} 33^{\prime} \mathrm{W}$ e altitude de $600 \mathrm{~m}$. O clima da região caracteriza-se por ser quente e úmido no verão e frio e seco no inverno. O total de chuvas no verão, comumente, excede $200 \mathrm{~mm}$ com chuvas quase que diárias. Já no inverno, a precipitação é bem reduzida e nos meses de junho a agosto a precipitação é freqüentemente inferior a $20 \mathrm{~mm}$ (Fisch, 1995). O ano hidrológico inicia-se em agosto e termina em julho do ano seguinte, com $42 \%$ da precipitação ocorrendo nos meses de dezembro a fevereiro. A precipitação média anual para o período de 1983 a 1991 foi de 1.350 mm (Fisch, 1995).

O solo da área em estudo corresponde ao Latossolo Vermelho-Amarelo (Embrapa, 1999) e as suas características químicas e físico-hídricas encontram-se nas Tabelas 1 e 2, respectivamente. A capacidade máxima de armazenamento do solo $(30 \mathrm{~mm})$ foi calculada de acordo com as propriedades físico-hídricas do solo para a profundidade de $25 \mathrm{~cm}$. Os valores de umidade na Capacidade de Campo $\left(\mathrm{CC}=0,34 \mathrm{~cm}^{3} \mathrm{~cm}^{-3}\right)$, Ponto de Murchamento Permanente (PMP $=0,218 \mathrm{~cm}^{3} \mathrm{~cm}^{-3}$ ) constantes da Tabela 2, foram determinados na curva de retenção da água no solo (Figura 1) para os potenciais de $100 \mathrm{cca}$ (cm de coluna de água) e 15.000 cca, respectivamente. A reserva de água no solo foi calculada em $60 \%$ da capacidade máxima de armazenamento (Doorembos; Kassam, 1994). Os valores de coeficiente de cultura (kc) para cálculo da evapotranspiração da cultura do trigo para os diferentes estádios foram baseados em dados recomendados pela FAO (Doorembos; Kassam, 1994) e encontram-se na Tabela 3. Para o Coeficiente de Tanque Classe A (kp) foi adotado o valor de 0,75 com base nos dados do posto meteorológico da UNITAU, localizado ao lado da área experimental.

Tabela 1. Características químicas do solo da área experimental.

\begin{tabular}{|c|c|c|c|c|c|c|c|}
\hline \multirow{3}{*}{ Ano } & pH & MO & $\mathbf{P}$ & $\mathbf{K}$ & $\mathrm{Ca}$ & Mg & \multirow{2}{*}{$\mathbf{H}+\mathbf{A I}$} \\
\hline & & & & \multirow{2}{*}{\multicolumn{4}{|c|}{$\begin{array}{c}- \\
-\end{array}$}} \\
\hline & $\mathrm{CaCl}_{2}$ & $g \mathrm{dm}^{-3}$ & $\mathrm{mg} \mathrm{dm}^{-3}$ & & & & \\
\hline 1999 & 4,4 & 18,3 & 9,3 & 2,8 & 14,7 & 5,2 & 39,2 \\
\hline 2000 & 5,4 & 18,0 & 12,0 & 4,5 & 23,0 & 14,0 & 24,0 \\
\hline
\end{tabular}

Tabela 2. Características físico-hídricas do solo (CC - Capacidade de Campo, PMP - Ponto de Murcha Permanente, $\mathrm{p}$ = porosidade, ds - densidade do solo, dp - densidade das partículas, VIB Velocidade de Infiltração Básica, Areia, Silte e Argila) da área experimental.

\begin{tabular}{|c|c|c|c|c|c|c|c|c|}
\hline CC & $\begin{array}{l}\text { PMP } \\
\mathrm{cm}^{3} \mathrm{~cm}\end{array}$ & $\mathbf{p}$ & ds & dp & $\begin{array}{c}\text { VIB } \\
\mathbf{m m ~ h}^{-1}\end{array}$ & Areia & $\begin{array}{l}\text { Silte } \\
\mathrm{g} \mathrm{dm}^{-}\end{array}$ & Argila \\
\hline 0,34 & 0,218 & 0,556 & 1,4 & 2,7 & 7,2 & 635 & 182 & 183 \\
\hline
\end{tabular}

Tabela 3. Coeficiente de cultura (kc) para diferentes períodos de desenvolvimento da cultura do trigo.

\begin{tabular}{cccccc}
\hline $\mathbf{k c}$ & $\mathbf{0 , 3}$ & $\mathbf{0 , 7}$ & $\mathbf{1 , 1}$ & $\mathbf{0 , 7}$ & $\mathbf{0 , 2}$ \\
\hline Período (dias) & $1-17$ & $18-58$ & $58-88$ & $89-122$ & $123-134$ \\
\hline
\end{tabular}


RUDORFF, B. F. T.; MOREIRA, A. M.; TARGA M. S.; FREITAS, J. G. Efeitos da irrigação e do nitrogênio na produtividade do trigo. Ambi-Agua, Taubaté, v. 1, n. 1, p. 48-60, 2006. (doi:10.4136/ambi-agua.5)

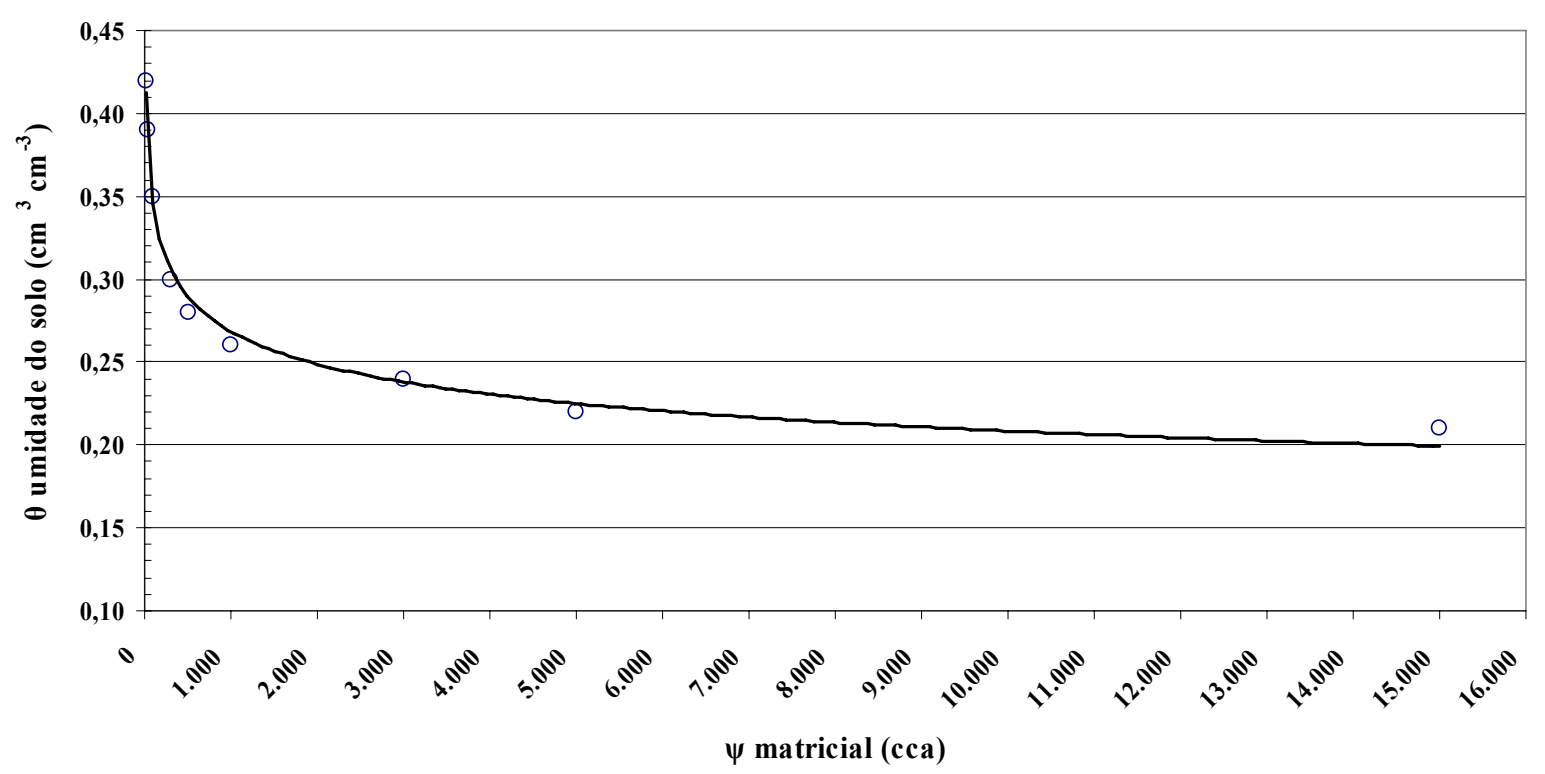

Figura 1. Curva de retenção de água no solo da área experimental.

O delineamento estatístico foi de blocos ao acaso, em esquema de parcelas sub subdivididas, com 4 repetições. Os tratamentos de irrigação foram aplicados nas parcelas de trigo de acordo com o seguinte protocolo: $100 \%$, 50\% e $25 \%$ da água evapotranspirada. Para o cálculo da lâmina de água a ser reposta nas parcelas, em função da evapotranspiração da cultura do trigo, empregou-se o manejo de irrigação recomendado pelo Boletim Técnico 167 (IAC, 1999) que tem por base a evaporação do tanque classe A. Para a decisão do momento de irrigação e da quantidade de água a ser aplicada foi feito um balanço hídrico diário. $\mathrm{O}$ sistema de irrigação foi instalado a cada ano logo após a semeadura e até o período de $\cong 40$ dias após emergência foi mantida a demanda de $100 \%$ da evapotranspiração da cultura para todas as parcelas, irrigando-se toda vez que cerca de $23 \%$ da capacidade de armazenamento do solo fosse consumido. Após, as parcelas com $50 \%$ e $25 \%$ da água evapotranspirada foram cobertas com lonas de plástico (Figura 2) durante os eventos de precipitação. A irrigação por aspersão foi aplicada com aspersor do tipo setorial de modo a atingir cada parcela específica, sempre no sentido de repor a água evapotranspirada no período, determinando-se a lâmina a ser reposta para que no tratamento de $100 \%$ o armazenamento de água no solo voltasse a 30 mm. A partir daí foram calculadas e aplicadas as lâminas correspondentes a 50\% e 25\% da evapotranspiração. Nesse período, a irrigação foi feita toda vez que a água equivalente a $40 \%$ da capacidade de armazenamento do solo fosse consumida.

Os cultivares IAC-289 e IAC-24 foram semeados nas sub-parcelas do experimento nos dias $1^{\circ}$ de maio de 1999 e 29 de maio de 2000. Os tratamentos de nitrogênio (N) foram aplicados nas sub sub-parcelas em quatro doses de $\mathrm{N}\left(0,60,90\right.$ e $120 \mathrm{~kg}$ de $\mathrm{N} \mathrm{ha}^{-1}$ ), utilizando-se uréia como fonte de $\mathrm{N}$. Um terço do $\mathrm{N}$ foi aplicado no sulco de semeadura junto

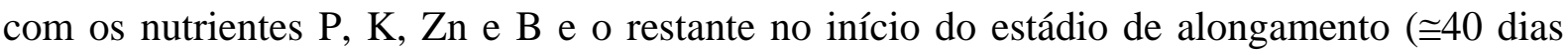
após a emergência das plantas, segundo recomendam Camargo et al. (1988).

A semeadura foi realizada por meio da semeadora de parcela de trigo do Centro de Plantas Graníferas do Instituto Agronômico de Campinas. O espaçamento foi de $17 \mathrm{~cm}$ entre linhas, com aproximadamente 80 sementes viáveis por metro linear. A adubação de macro e micronutrientes teve por base a análise de solo da área experimental e a tabela de recomendação de adubação para a cultura do trigo no Estado de São Paulo (IAC, 1999). Não foi necessária a aplicação de defensivos agrícolas. No levantamento da fertilidade e da física do solo foi verificado que não havia a necessidade de se realizar uma subsolagem. A 
descrição dos estádios fenológicos do trigo foi feita utilizando-se a escala decimal de crescimento dos cereais segundo Zadoks et al. (1974) citado em Scheeren (1986). A colheita do trigo foi realizada na parte central de cada unidade experimental, desprezando-se duas linhas de cada lado e 0,5 m no comprimento, para evitar o efeito de bordadura. Os valores de produtividade de grãos, fitomassa seca da parte aérea, índice de colheita, massa de mil grãos, número de sementes por espiga, tamanho de espigas, número de espiguetas desenvolvidas e não desenvolvidas foram analisados utilizando-se procedimentos de análise de variância adequados para um delineamento experimental em blocos com sub sub-parcelas. As diferenças entre tratamentos foram avaliadas pelo teste estatístico de Duncan $(\alpha=0,05)$.
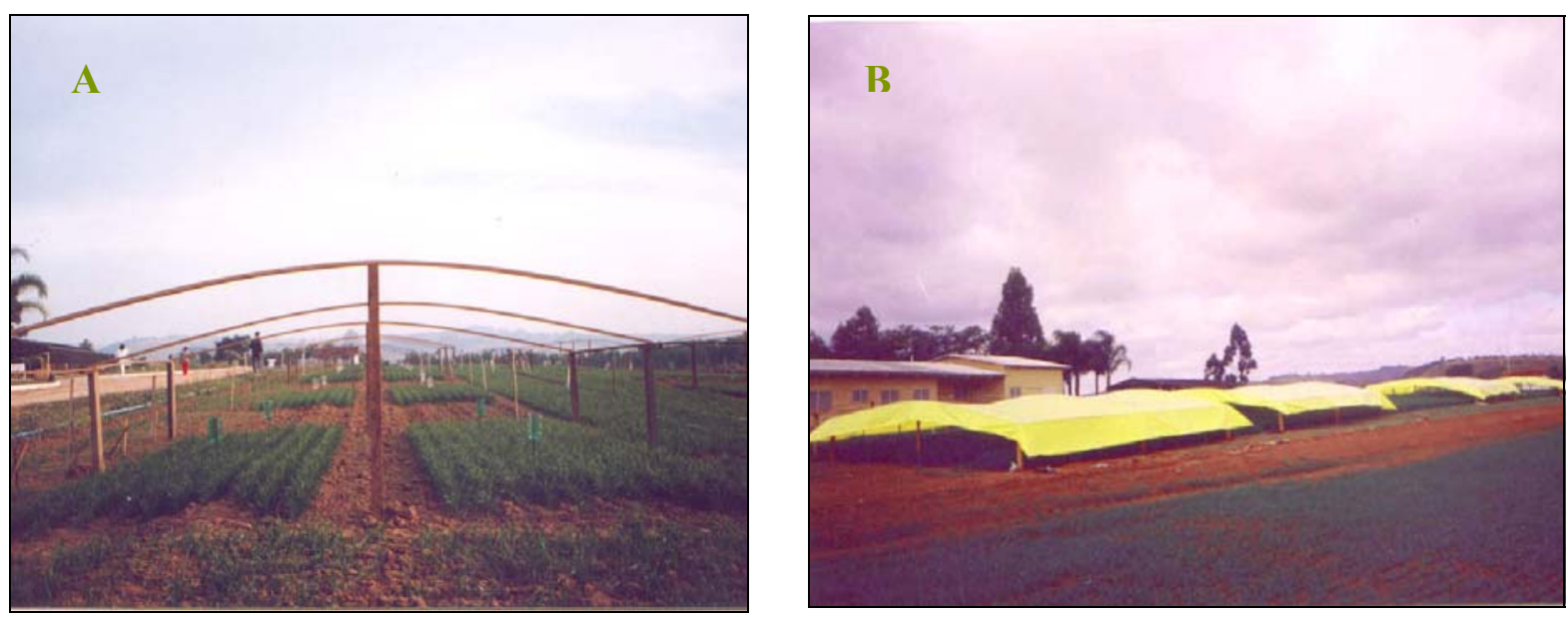

Figura 2. (A) Suporte de madeira para cobrir as parcelas com lonas plásticas e (B) vista panorâmica das parcelas cobertas com lonas plásticas durante evento de precipitação.

\section{RESULTADOS E DISCUSSÃO}

As análises estatísticas das características agronômicas para as safras de 1999 e 2000 estão apresentadas nas Tabelas 4 e 5, respectivamente. Nota-se nessas tabelas que não houve efeito significativo $(\alpha \leq 0,05)$ para a interação irrigação vs. cultivar vs. nitrogênio para qualquer uma das características analisadas. Apenas em 1999 (Tabela 4) foi observado um efeito significativo na produtividade de grãos e na fitomassa para a interação irrigação vs. nitrogênio. Isso indica que pelo menos um dos níveis de irrigação apresenta uma resposta diferenciada às doses de nitrogênio. Ainda em 1999, foi observado um efeito significativo na produtividade de grãos e na massa de 1.000 grãos para a interação cultivar vs. nitrogênio indicando que os dois cultivares apresentaram uma resposta diferenciada às doses de nitrogênio (Tabela 4).

A irrigação teve efeito significativo sobre a produtividade de grãos e fitomassa nos dois anos safra (Tabelas 4 e 5). Em 1999, a irrigação teve efeito mais pronunciado do que em 2000 e a produtividade de grãos foi reduzida em 38,3\% e 54,7\% para os níveis de 50\% e $25 \%$ da evapotranspiração, respectivamente (Tabela 4). Também houve diferença significativa entre esses dois níveis de irrigação, havendo uma redução na produtividade de grãos de 26,6\% para o nível de irrigação de $25 \%$ em relação ao nível de $50 \%$ da evapotranspiração (Tabela 4). Na safra de 2000, a produtividade de grãos foi reduzida em 33,5\% e 37,4\% para os níveis de irrigação de $50 \%$ e $25 \%$ da evapotranspiração, respectivamente (Tabela 5). Contudo não foi observada uma diferença significativa para produtividade de grãos entre os níveis de 50\% e 25\% da evapotranspiração (Tabela 5).

A redução na produtividade de grãos em função da irrigação observada nos dois anos safra não pode ser explicada pelas diversas características agronômicas medidas neste estudo 
e apresentadas nas Tabelas 4 e 5. Por exemplo, em 1999, a massa de 1.000 grãos foi significativamente reduzida para o nível de irrigação de $25 \%$ da evapotranspiração, enquanto que a produtividade de grãos foi reduzida tanto a $25 \%$ quanto a $50 \%$ da evapotranspiração (Tabela 4). Outra característica, como tamanho da espiga, apresenta diferença significativa apenas em 1999. No ano safra de 1999, nota-se que o número de espiguetas desenvolvidas foi significativamente reduzido quando as plantas estiveram submetidas aos níveis de irrigação de $50 \%$ e $25 \%$ da evapotranspiração, explicando parte da redução observada na produtividade de grãos (Tabela 4).

Tabela 4. Efeitos dos fatores irrigação, cultivar e nitrogênio sobre as características agronômicas do trigo no ano de 1999.

\section{A R A C T E R Í S T I C A S A G R O NÔMI C A S}

\begin{tabular}{|c|c|c|c|c|c|c|c|c|c|c|}
\hline \multicolumn{3}{|c|}{ FATORES } & $\begin{array}{l}\text { Produti- } \\
\text { vidade } \\
\left(\mathrm{g} \mathrm{m}^{-2}\right)\end{array}$ & $\begin{array}{l}\text { Fito- } \\
\text { massa } \\
\left(\mathrm{g} \mathrm{m}^{-2}\right)\end{array}$ & $\begin{array}{l}\text { Índice } \\
\text { de } \\
\text { Colheita } \\
(\%)\end{array}$ & $\begin{array}{l}\text { Massa } \\
\text { mil } \\
\text { grãos } \\
\text { (g) }\end{array}$ & $\begin{array}{c}\text { grãos } \\
\text { espiga } \\
1 \\
\left(\mathrm{~N}^{\circ}\right)\end{array}$ & $\begin{array}{c}\text { Tamanho } \\
\text { espiga } \\
\text { (cm) }\end{array}$ & $\begin{array}{c}\text { Espigueta } \\
\text { desenvol. } \\
\left(\mathrm{N}^{0}\right)\end{array}$ & $\begin{array}{l}\text { Espigueta } \\
\text { não } \\
\text { desenvol. } \\
\left(\mathrm{N}^{\circ}\right)\end{array}$ \\
\hline Irrigação & Cultivar & Nitrogênio & & & & & & & & \\
\hline $100 \%$ & - & - & 354,3 a & 785,1 a & 45,4 & $40,1 \mathrm{a}$ & 25,4 a & 6,5 a & $13,8 \mathrm{a}$ & $3,9 \mathrm{~b}$ \\
\hline $50 \%$ & - & - & 218,5 b & $468,9 \mathrm{~b}$ & 47,3 & 39,5 a & $18,3 \mathrm{~b}$ & $5,8 \mathrm{~b}$ & $11,4 \mathrm{ab}$ & 5,4 a \\
\hline $25 \%$ & - & - & 160,4 c & $342,4 \mathrm{c}$ & 47,1 & $33,8 \mathrm{~b}$ & $15,0 \mathrm{~b}$ & $5,4 \mathrm{~b}$ & $10,5 \mathrm{~b}$ & 6,0 a \\
\hline- & IAC-24 & - & 235,6 & 535,6 & $44,2 \mathrm{~b}$ & 39,1 & 19,6 & 6,0 & 11,9 & 5,2 \\
\hline - & IAC-289 & - & 243,3 & 507,9 & 48,9 a & 36,5 & 19,8 & 5,9 & 11,9 & 5,0 \\
\hline \multirow{2}{*}{-} & - & 0 kg.ha ${ }^{-1}$ & 207,9 c & $458,4 \mathrm{c}$ & $45,2 \mathrm{~b}$ & 7,5 & 20,3 & 6,1 & 12,0 & 5,2 \\
\hline & - & 60 kg.ha ${ }^{-1}$ & $240,0 \mathrm{~b}$ & $511,8 \mathrm{~b}$ & $48,3 \mathrm{a}$ & 38,6 & 19,4 & 5,9 & 11,9 & 5,2 \\
\hline- & & 90 kg.ha ${ }^{-1}$ & $242,7 \mathrm{~b}$ & $527,0 \mathrm{~b}$ & $47,1 \mathrm{ab}$ & 37,7 & 19,8 & 6,0 & 12,1 & 5,2 \\
\hline \multirow[t]{2}{*}{-} & - & $120 \mathrm{~kg} \cdot \mathrm{ha}^{-1}$ & 267,7 a & 587,4 a & $46,1 \mathrm{ab}$ & 37,4 & 19,2 & 5,8 & 11,7 & 4,8 \\
\hline & & & \multicolumn{8}{|c|}{ Análise de variância, valores de $\mathbf{F} U$} \\
\hline \multicolumn{3}{|l|}{ Irrigação } & $35,3^{* *}$ & $43,1^{* *}$ & 2,8 NS & $112,1^{* *}$ & $25,5^{* *}$ & $9,8^{*}$ & 4,1 NS & $20,3 * *$ \\
\hline \multicolumn{3}{|l|}{ Cultivar } & $0,1 \mathrm{NS}$ & 4,2 NS & $29,9 * *$ & 2,7 NS & $0,2 \mathrm{NS}$ & $0,0 \mathrm{NS}$ & $0,0 \mathrm{NS}$ & $1,8 \mathrm{NS}$ \\
\hline \multicolumn{3}{|l|}{ Nitrogênio } & $13,6^{* *}$ & $14,4^{* *}$ & 1,8 NS & $0,2 \mathrm{NS}$ & $0,4 \mathrm{NS}$ & 1,1 NS & $0,5 \mathrm{NS}$ & $1,4 \mathrm{NS}$ \\
\hline \multicolumn{3}{|c|}{ Irrigação x Cultivar } & $2,3 \mathrm{NS}$ & $1,5 \mathrm{NS}$ & $0,7 \mathrm{NS}$ & $0,3 \mathrm{NS}$ & $5,2^{*}$ & $3,1 \mathrm{NS}$ & $7,4^{*}$ & 3,3 NS \\
\hline \multicolumn{3}{|c|}{ Irrigação x Nitrogênio } & $2,6^{*}$ & $5,2 * *$ & $0,9 \mathrm{NS}$ & $0,7 \mathrm{NS}$ & $0,9 \mathrm{NS}$ & $0,8 \mathrm{NS}$ & $2,0 \mathrm{NS}$ & $1,2 \mathrm{NS}$ \\
\hline \multicolumn{3}{|c|}{ Cultivar x Nitrogênio } & $2,8^{*}$ & $1,5 \mathrm{NS}$ & $0,8 \mathrm{NS}$ & $2,9 *$ & $1,6 \mathrm{NS}$ & 2,1 NS & $1,7 \mathrm{NS}$ & $0,3 \mathrm{NS}$ \\
\hline \multicolumn{3}{|c|}{ IrrigaçãoxCultivarxNitrogênio } & $0,8 \mathrm{NS}$ & 0,4 NS & $0,1 \mathrm{NS}$ & $1,3 \mathrm{NS}$ & $1,5 \mathrm{NS}$ & $0,6 \mathrm{NS}$ & $0,6 \mathrm{NS}$ & $1,9 \mathrm{NS}$ \\
\hline \multicolumn{3}{|c|}{ Coeficiente de Variação, \% } & 13,3 & 12,9 & 9,6 & 13,8 & 18,6 & $8,6 \mathrm{NS}$ & 10,4 & 15,6 \\
\hline
\end{tabular}

NS, não significativo no nível de $P \leq 0,05 ; *$, **, significativo nos níveis de 0,05 e 0,01, respectivamente. Médias com letras iguais não diferem significativamente no nível de 5\% pelo teste de Duncan. 
Tabela 5. Efeitos dos fatores irrigação, cultivar e nitrogênio sobre as características agronômicas do trigo no ano de 2000.

$$
\text { C A R A C TERÍS TIC A S A GRON ÔMI C A S }
$$

\begin{tabular}{|c|c|c|c|c|c|c|c|c|c|c|}
\hline \multicolumn{3}{|c|}{ FATORES } & \multirow[t]{2}{*}{$\begin{array}{l}\text { Produti- } \\
\text { vidade } \\
\left(\mathrm{g} \mathrm{m}^{-2}\right)\end{array}$} & \multirow[t]{2}{*}{$\begin{array}{l}\text { Fito- } \\
\text { massa } \\
\left(\mathrm{g} \mathrm{m}^{-2}\right)\end{array}$} & \multirow[t]{2}{*}{$\begin{array}{l}\text { Índice } \\
\text { de } \\
\text { Colheita } \\
(\%)\end{array}$} & \multirow[t]{2}{*}{$\begin{array}{l}\text { Massa } \\
\text { mil } \\
\text { grãos } \\
\text { (g) }\end{array}$} & \multirow[t]{2}{*}{$\begin{array}{c}\text { grãos } \\
\text { espiga } \\
\frac{1}{\left(N^{\circ}\right)}\end{array}$} & \multirow[t]{2}{*}{$\begin{array}{c}\text { Tamanh } \\
\text { o espiga } \\
\text { (cm) }\end{array}$} & \multirow[t]{2}{*}{$\begin{array}{l}\text { Espigueta } \\
\text { desenvol. } \\
\left(\mathrm{N}^{\circ}\right)\end{array}$} & \multirow[t]{2}{*}{$\begin{array}{l}\text { Espigueta } \\
\text { não } \\
\text { desenvol. } \\
\left(\mathrm{N}^{\circ}\right)\end{array}$} \\
\hline$\underline{\text { Irrigação }}$ & $\underline{\text { Cultivar }}$ & Nitrogênio & & & & & & & & \\
\hline $100 \%$ & - & - & 413,6 a & 813,0 a & 51,0 & 38,8 & 29,9 & 7,4 & 14,3 & 3,1 a \\
\hline $50 \%$ & - & - & $275,0 \mathrm{~b}$ & $534,6 \mathrm{~b}$ & 51,4 & 42,4 & 31,6 & 7,6 & 14,2 & $3,0 a b$ \\
\hline $25 \%$ & - & - & 258,8 b & $513,6 \mathrm{~b}$ & 50,4 & 38,7 & 33,8 & 7,6 & 14,7 & $2,7 \mathrm{~b}$ \\
\hline- & IAC-24 & - & 330,0 a & 626,9 & $47,9 \mathrm{~b}$ & 40,6 & 31,8 & 7,5 & 14,4 & 2,9 \\
\hline- & IAC-289 & - & 301,6 b & 613,9 & 53,9 a & 39,4 & 31,8 & 7,6 & 14,4 & 3,0 \\
\hline- & - & 0 kg.ha ${ }^{-1}$ & 310,4 & 605,5 & 51,4 & 40,7 & 32,0 & 7,5 & 14,2 & $2,9 \mathrm{ab}$ \\
\hline & - & 60 kg.ha ${ }^{-1}$ & 320,9 & 627,6 & 50,8 & 39,0 & 31,8 & 7,5 & 14,5 & $2,8 \mathrm{~b}$ \\
\hline & & 90 kg.ha ${ }^{-1}$ & 307,2 & 614,4 & 50,3 & 39,9 & 31,2 & 7,5 & 14,2 & 3,3 a \\
\hline \multirow[t]{2}{*}{ _ } & - & 120kg.ha ${ }^{-1}$ & 324,5 & 634,0 & 51,3 & 40,3 & 32,0 & 7,6 & 14,6 & $2,8 \mathrm{~b}$ \\
\hline & & & \multicolumn{8}{|c|}{ Análise de variância, valores de F U } \\
\hline \multicolumn{3}{|l|}{ Irrigação } & $32,0 * *$ & $41,3^{* *}$ & $0,8 \mathrm{NS}$ & $3,7 \mathrm{NS}$ & $1,4 \mathrm{NS}$ & 0,3 NS & $1,1 \mathrm{NS}$ & $4,2 \mathrm{NS}$ \\
\hline \multicolumn{3}{|l|}{ Cultivar } & $5,4 *$ & 0,4 NS & $80,3^{* *}$ & $4,0 \mathrm{NS}$ & $0,0 \mathrm{NS}$ & 4,2 NS & 0,2 NS & 0,2 NS \\
\hline \multicolumn{3}{|l|}{ Nitrogênio } & 0,7 NS & $0,5 \mathrm{NS}$ & 0,3 NS & $0,7 \mathrm{NS}$ & $0,2 \mathrm{NS}$ & $0,2 \mathrm{Ns}$ & $0,5 \mathrm{NS}$ & $2,7 *$ \\
\hline \multicolumn{3}{|c|}{ Irrigação x Cultivar } & $0,1 \mathrm{NS}$ & 0,9 NS & $5,3^{*}$ & $0,4 \mathrm{NS}$ & $4,6^{*}$ & 2,0 NS & $0,5 \mathrm{NS}$ & 1,1 NS \\
\hline \multicolumn{3}{|c|}{ Irrigação x Nitrogênio } & $0,5 \mathrm{NS}$ & 0,4 NS & $1,4 \mathrm{NS}$ & $0,2 \mathrm{NS}$ & 0,3 NS & 0,8 NS & 0,7 NS & 0,9 NS \\
\hline \multicolumn{3}{|c|}{ Cultivar x Nitrogênio } & $1,0 \mathrm{NS}$ & $1,0 \mathrm{NS}$ & 0,4 NS & $0,8 \mathrm{NS}$ & $0,5 \mathrm{NS}$ & 0,9 NS & 0,4 NS & 1,9 NS \\
\hline \multicolumn{3}{|c|}{ IrrigaçãoxCultivarxNitrogênio } & $1,6 \mathrm{NS}$ & 0,3 Ns & 1,9 NS & 0,5 NS & 0,6 NS & $1,0 \mathrm{NS}$ & 0,9 NS & $0,3 \mathrm{NS}$ \\
\hline \multicolumn{3}{|c|}{ Coeficiente de Variação, \% } & 14,8 & 13,8 & 9,5 & 10,2 & 15,4 & 8,8 & 9,2 & 23,0 \\
\hline
\end{tabular}

NS, não significativo no nível de $P \unlhd 0,05$; *, **, significativo nos níveis de 0,05 e 0,01, respectivamente. Médias com letras iguais não diferem significativamente no nível de 5\% pelo teste de Duncan.

Durante as estações de crescimento do trigo, as temperaturas médias máximas e mínimas foram de $11,0{ }^{\circ} \mathrm{C}$ e $26,3{ }^{\circ} \mathrm{C}$ em 1999 e de $8,8{ }^{\circ} \mathrm{C}$ e $27,6{ }^{\circ} \mathrm{C}$ em 2000 , respectivamente. Isso indica que os anos foram muito semelhantes entre si no que se refere à temperatura. $\mathrm{A}$ principal diferença entre os anos foi a disponibilidade de água, conforme pode ser observado no balanço hídrico apresentado nas Figuras 3 e 4 para os anos de 1999 e 2000, respectivamente. A Figura 5 apresenta a síntese do balanço hídrico para os anos de 1999 e 2000. Na Figura 3, referente ao cultivo de 1999, observa-se que houve uma maior concentração de chuva até os 50 dias iniciais de cultivo, enquanto que no ano de 2000 (Figura 4) praticamente não ocorreu chuva na fase inicial do cultivo com a maior concentração de chuva durante os 50 dias finais do cultivo. Foi observado que não houve diferença significativa entre os tratamentos de irrigação de $50 \%$ e $25 \%$ da evapotranspiração em 2000 , para nenhuma das características agronômicas; muito embora, a Figura 5 apresente diferenças no balanço hídrico para esses dois tratamentos. Isso pode ser atribuído ao fato de que durante os 40 dias iniciais, todas as parcelas receberam a mesma quantidade de água e quando elas foram submetidas aos tratamentos de irrigação o solo pode ter fornecido água de maior profundidade (Fisch, 1995). Somado a isso, a precipitação ocorrida nos 50 dias finais do 
RUDORFF, B. F. T.; MOREIRA, A. M.; TARGA M. S.; FREITAS, J. G. Efeitos da irrigação e do nitrogênio na produtividade do trigo. Ambi-Agua, Taubaté, v. 1, n. 1, p. 48-60, 2006. (doi:10.4136/ambi-agua.5)

1999 - Reposição 100\%

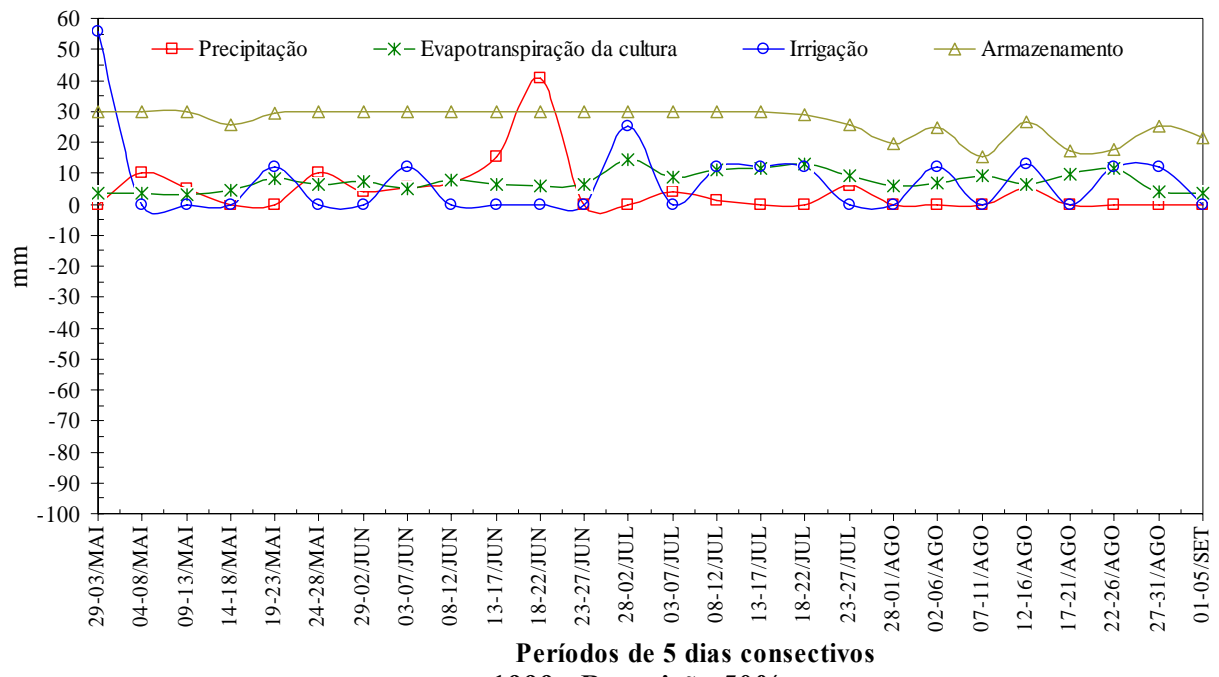

1999 - Reposição 50\%

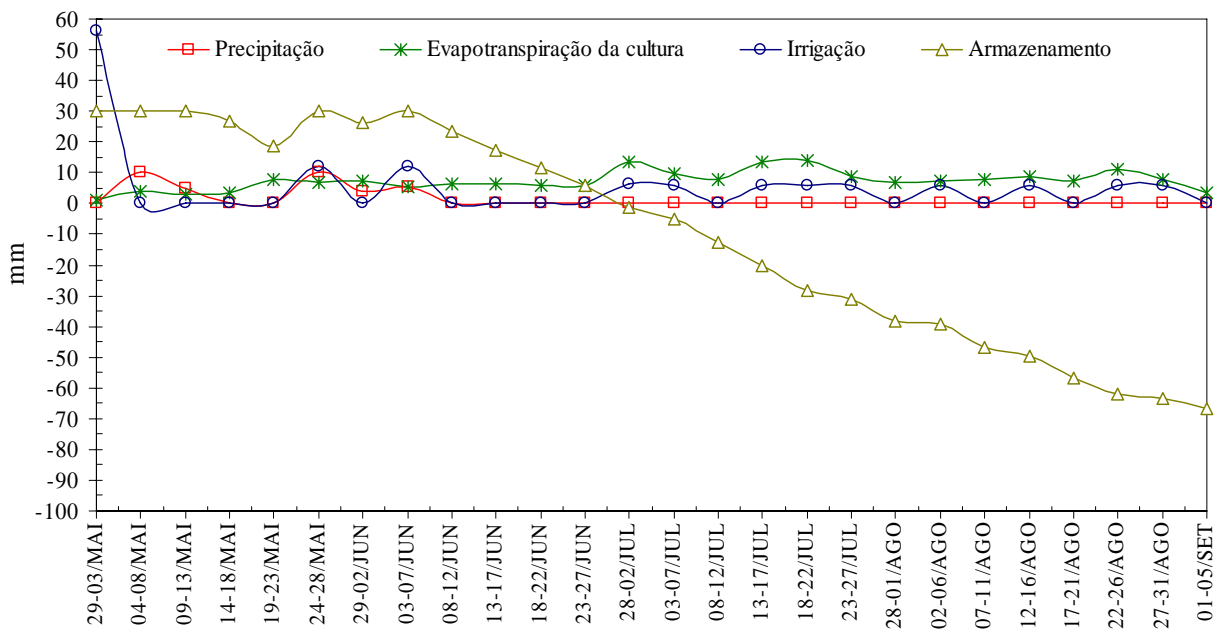

Períodos de 5 dias consecutivos

1999 - Reposição $25 \%$

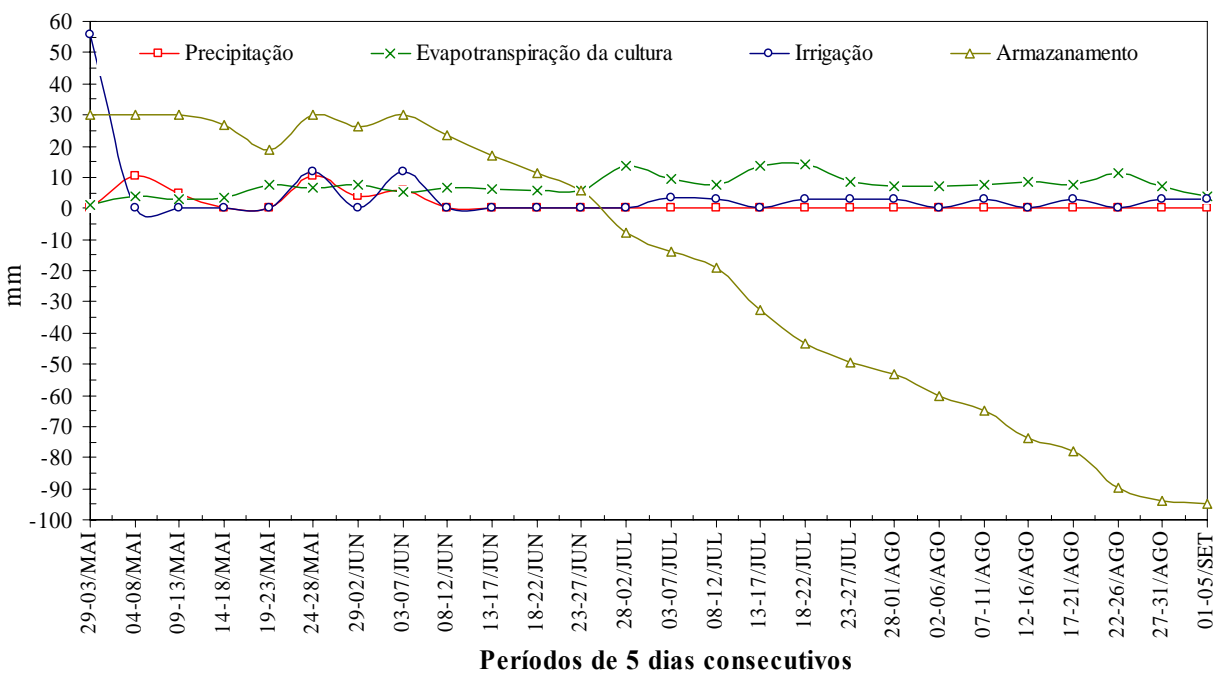

Figura 3. Balanço hídrico para os três tratamentos de irrigação (100\%, $50 \%$ e 25\% da evapotranspiração) da cultura trigo no ano de 1999. 
RUDORFF, B. F. T.; MOREIRA, A. M.; TARGA M. S.; FREITAS, J. G. Efeitos da irrigação e do nitrogênio na produtividade do trigo. Ambi-Agua, Taubaté, v. 1, n. 1, p. 48-60, 2006. (doi:10.4136/ambi-agua.5)

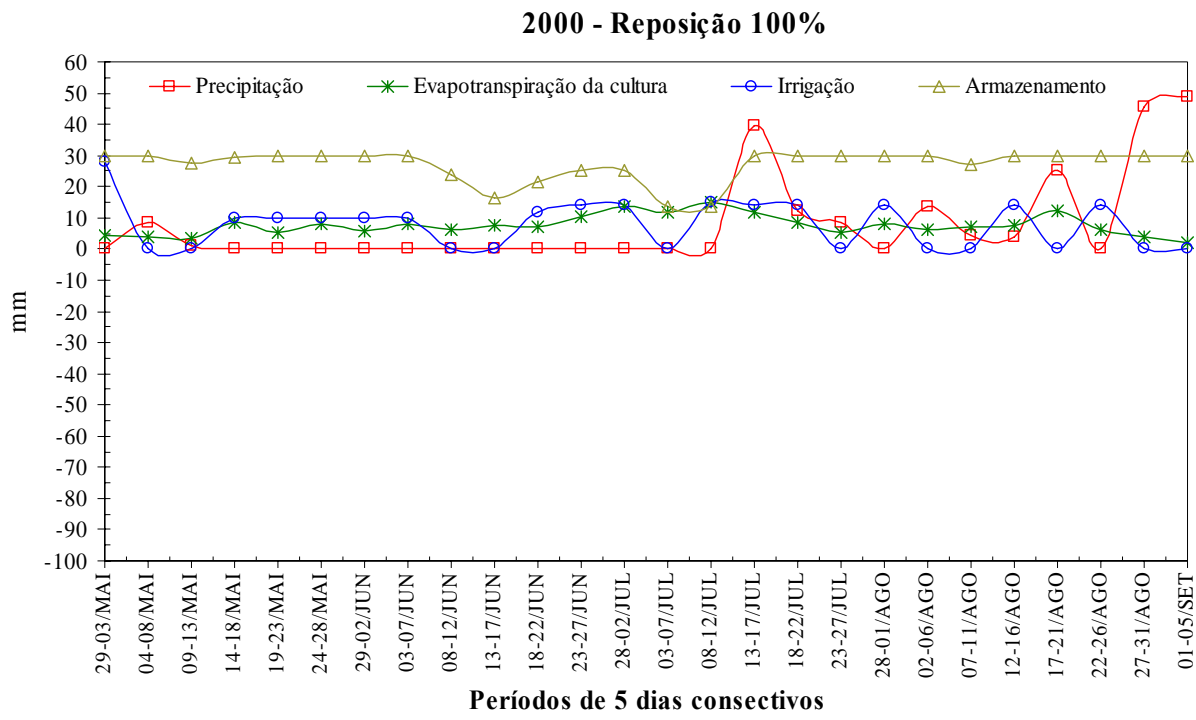

2000 - Reposição 50\%

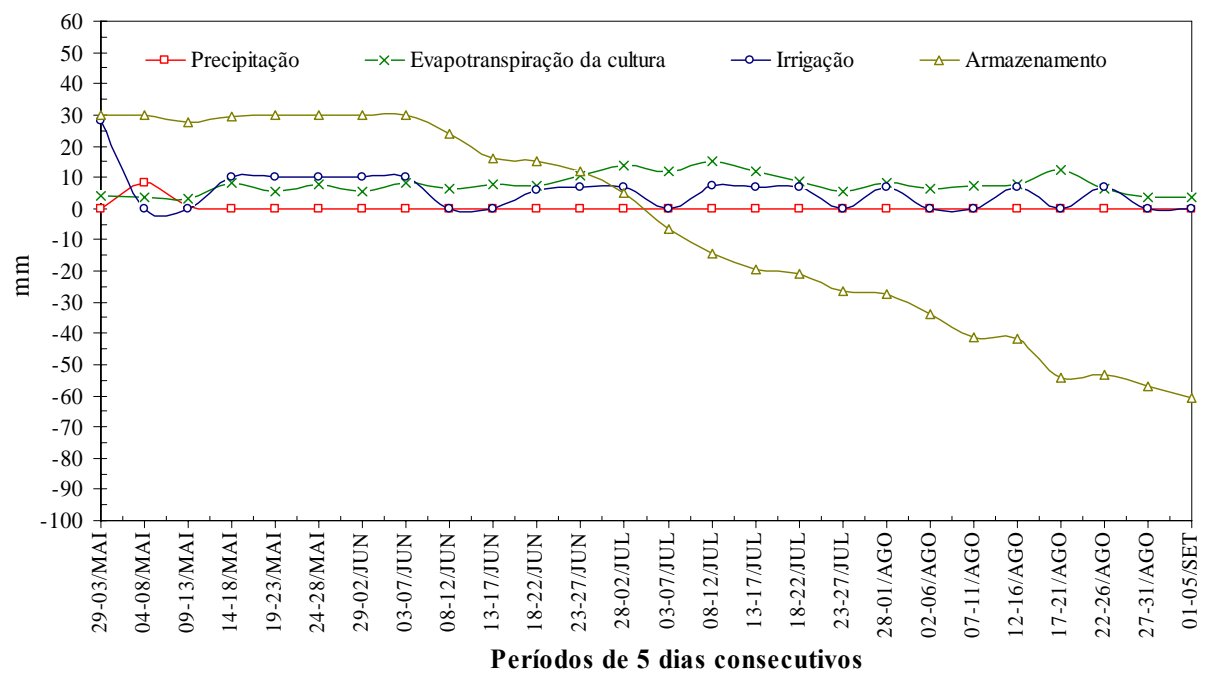

2000 - Reposição $25 \%$

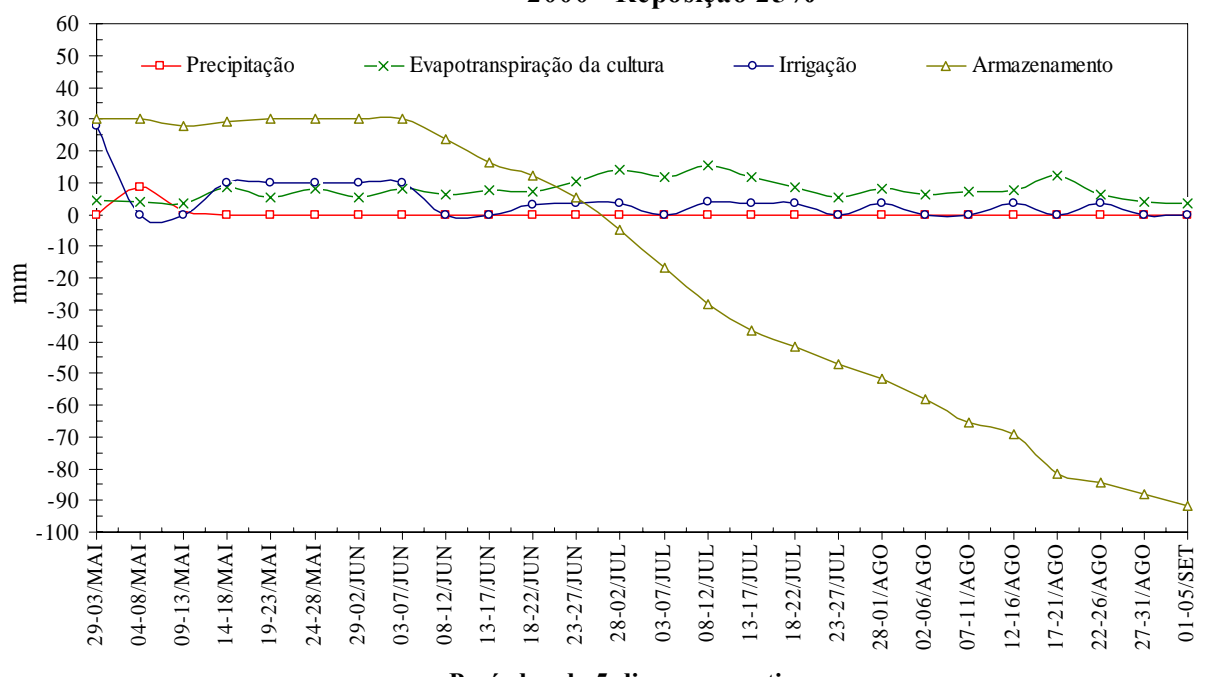

Figura 4. Balanço hídrico para os três tratamentos de irrigação (100\%, 50\% e

$25 \%$ da evapotranspiração) da cultura trigo no ano de 2000.

cultivo pode ter afetado as parcelas com os tratamentos de $50 \%$ e $25 \%$ da evapotranspiração devido à falha na cobertura dessas parcelas com as lonas plásticas, permitindo alguma infiltração de água da chuva durante os eventos de precipitação em 2000. 
Com base nos resultados observados nos dois anos do experimento, pode-se afirmar que a redução na disponibilidade de água para as plantas de trigo, para os níveis de $50 \%$ e $25 \%$ da evapotranspiração, reduz significativamente a produtividade de grãos. Observa-se ainda que de 1999 para 2000 houve um aumento na produtividade de grãos, fitomassa da parte aérea, índice de colheita, massa de 1.000 grãos, número de grãos por espiga, tamanho das espigas, número de espiguetas desenvolvidas, bem como uma redução no número de espiguetas não desenvolvidas. Todavia, em 2000 não houve um aumento significativo da produtividade de grãos quando se passa de $25 \%$ para $50 \%$ da evapotranspiração (Tabela 5). É importante salientar que até o período de $\cong 40$ dias após emergência foi mantida a demanda evaporativa de $100 \%$ para todas as parcelas, não havendo déficit de água no estádio próximo à floração.
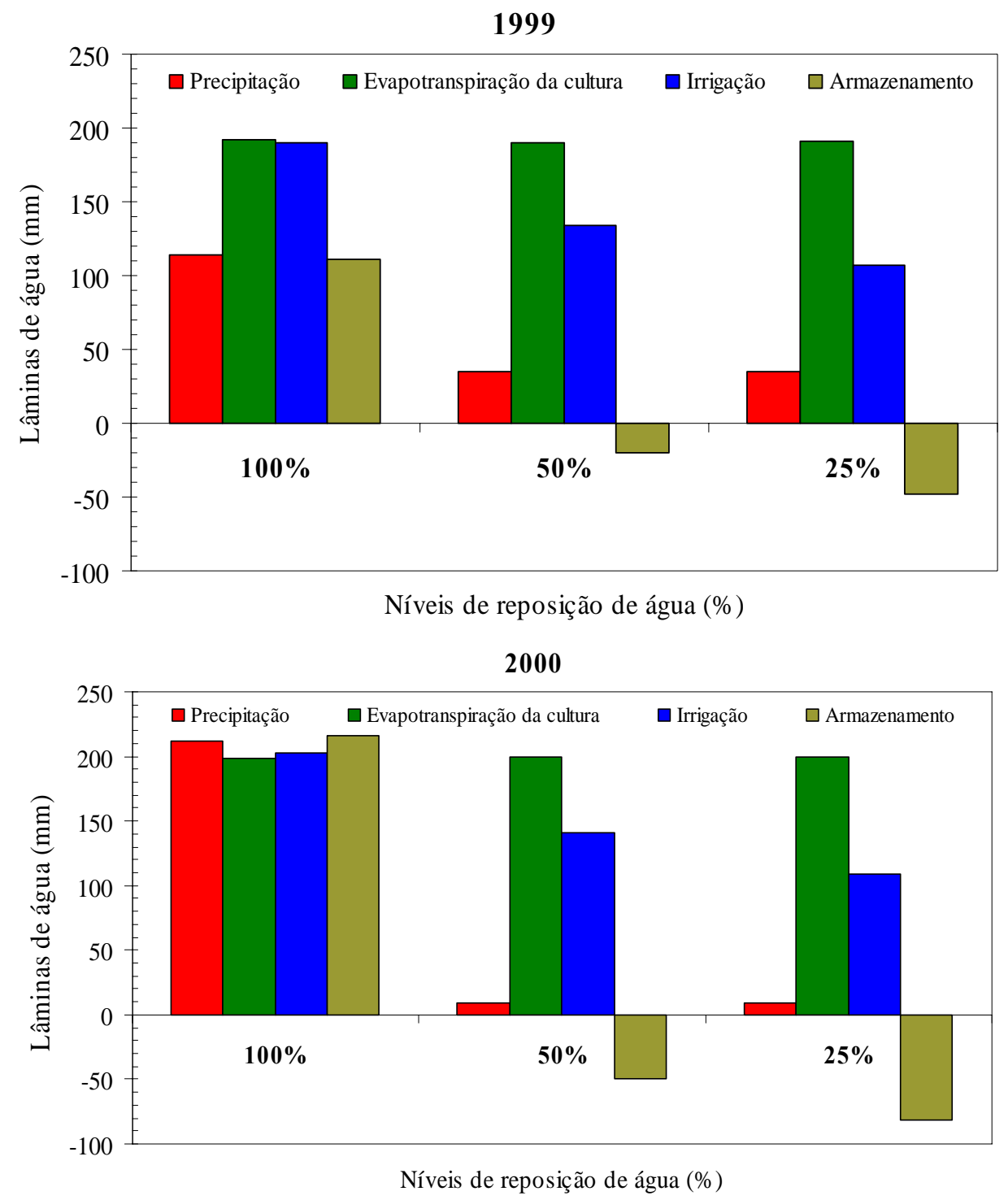

Figura 5. Síntese do balanço hídrico da cultura do trigo para os níveis de reposição de água de 100, 50 e $25 \%$ da evapotranspiração da cultura para os anos de 1999 e 2000.

A diferença entre os cultivares aqui estudados não fica evidente quanto à produtividade de grãos uma vez que somente em 2000 o cultivar IAC-24 apresentou melhor desempenho quando comparado com o cultivar IAC-289. Moreira et al. (2005), num estudo comparativo entre genótipos de trigo também não observaram diferença na produtividade desses dois 
cultivares. A adubação nitrogenada apresentou efeito significativo somente no ano de 1999, quando a produtividade de grãos foi aumentada em $15,4 \%$ e $28,8 \%$ para as doses de 60 kg.ha-1 e $120 \mathrm{~kg} \cdot \mathrm{ha}^{-1}$ de $\mathrm{N}$, respectivamente.

Um aspecto interessante a ser destacado se refere à diferença nos valores de produtividade de grãos entre os anos 1999 e 2000 (Tabelas 4 e 5), quando foi observado um aumento de $16,7 \%, 25,8 \%$ e $61,3 \%$ na produtividade de grãos para as parcelas de trigo irrigado a $100 \%, 50 \%$ e $25 \%$ da evapotranspiração, respectivamente. Isso pode ser atribuído à melhoria nas características químicas do solo (Tabela 1) da área experimental realizada por meio de calagem e adubação corretiva, mostrando ainda que o maior benefício dessa melhoria sobre a produtividade de grãos, foi observado para os tratamentos com menor disponibilidade de água para a cultura.

\section{CONCLUSÃO}

No presente trabalho não foi observada uma interação significativa entre os fatores irrigação, cultivar e nitrogênio para qualquer uma das características analisadas. O efeito interativo entre irrigação e nitrogênio aparece apenas no ano de 1999, quando a produtividade e a fitomassa apresentaram efeito significativo para as doses de nitrogênio em função do nível de irrigação. A melhoria das características químicas do solo por meio de calagem e adubação corretiva aumentou significativamente a produtividade de grãos de trigo, particularmente, para os tratamentos com níveis de irrigação de $50 \%$ e $25 \%$ da evapotranspiração. A prática da irrigação se mostrou fundamental para obtenção de uma produtividade rentável da cultura do trigo na região do Vale do Paraíba durante o período seco da entressafra. O balanço hídrico é uma boa forma de racionalizar o uso da água na agricultura, contribuindo com a conservação ambiental.

\section{AGRADECIMENTOS}

À Fapesp (Fundação de Amparo à Pesquisa do Estado de São Paulo) pelo financiamento do projeto número 97/11075-7, que possibilitou a realização deste trabalho. Aos funcionários do Instituto Agronômico de Campinas, pelo apoio dado na condução do experimento, em especial os funcionários: José Norberto da Silva, Ismael dos Santos Ribeiro, Eurico Alves, Carlos Aparecido Fernandes e José Carlos de Andrade. Aos funcionários do Laboratório de Análise de Solo da Universidade de Agronomia de Taubaté (UNITAU), pela pronta colaboração nas análises físicas e químicas das amostras do solo da área experimental. Aos alunos do Departamento de Agronomia: José Flávio Corte e Leandro Massao Paes Aikawa pelo interesse, seriedade e dedicação na coleta e acompanhamento das análises dos dados dos experimentos. 


\section{REFERÊNCIAS}

BOARETTO, A. E.; SPOLIDORIO, E. S.; TRIBELIN, P. C. O.; MURAOKA, T.; FREITAS, J. G. et al. Optimizing nitrogen-fertilzer application to wheat under irigation. In: Optimizing nitrogen fertilizer application to irrigated wheat. Viena: International Atomic Energy Agency, 2000. p. 63-72. vol. 1. (ISSN 1011-4289).

CAMARGO, C. E. O. Adubação de trigo: IX - interpretação econômica dos resultados obtidos em experimentos com N, P, K e S, em latossolo roxo do estado de São Paulo. Bragantia, Campinas, v. 35, n. 1, p. 95-106, 1976.

CAMARGO, C. E. O.; FELICIO, J. C.; PETINELLI JR., A.; ROCHA JR., L. S. Adubação nitrogenada em cultura do trigo irrigado por aspersão no estado de São Paulo. Campinas: Instituto Agronômico, 1988. 26 p. (Boletim científico, 15).

DOOREMBOS, J.; KASSAM, A.H. Efeito da água no rendimento das culturas. In: GHEYI et al. (Trad.). Estudos FAO: irrigação e drenagem. Campina Grande: UFPB, 1994. (Boletim 33).

EMPRESA BRASILEIRA DE PESQUISA AGROPECUÁRIA (EMBRAPA). Centro Nacional de Pesquisas de Solos. Sistema brasileiro de classificação de solos. Rio de Janeiro: Embrapa Solos, 1999. 412 p.

. Departamento Técnico-Científico. Programa Nacional de Pesquisa de Trigo. Brasília: Embrapa-DID, 1981. 100p.

EPSTEIN, E. A. Aquisição de nitrogênio. In: MALAVOLTA, E. (Trad.). Nutrição mineral das plantas: princípios e perspectivas. Rio de Janeiro: Livros Técnicos e Científicos, 1975. p. 213-34.

FELICIO, J. C.; CAMARGO, C. E. O.; GERMANI, R.; GALLO, P. B.; PEREIRA, J. C. V. $\mathrm{N}$. A. et al. Influência do ambiente no rendimento e na qualidade de grãos de genótipos de trigo com irrigação por aspersão no estado de São Paulo. Bragantia, Campinas, v. 60, n. 2, p. 111-120, 2001.

FERrEIRA, P. A.; CARDOSO, A. A.; FERNANDES, B.; PARENTES, A. C. Efeito de diferentes níveis de tensão de umidade do solo sobre a produção de trigo. Revista Ceres, Viçosa, v. 20, p.129-135, 1973. (ISSN 1011-4289).

FISCH, G. Caracterização climática e balanço hídrico de Taubaté (SP). Revista Biociências, Taubaté, v.1, n. 1, p. 81-90. 1995.e

FRIZZONE, J. A.; MÉLlO JR., A. V.; FOLEGATTI, M. V.; BOTREL, T. A.. Efeito de diferentes níveis de irrigação e adubação nitrogenada sobre componentes de produtividade do trigo. Pesquisa Agropecuária Brasileira, Brasília, v. 31, n. 6, p. 425434, 1996.

FRIZZONE, J. A.; ZANINI, J. R.; PEREIRA, G. T.; RETTORE, P. R.. Efeito da freqüência e da lâmina de irrigação na produção de trigo (Triticum aestivum, L.). Ciência e Prática, Lavras, v. 9, n. 2, p. 198-207, 1985.

INSTITUTO AGRONÔMICO (IAC). Trigo: recomendações. 2. ed. Campinas: IAC, 1999. 100p. (Boletim Técnico, 167). 
MOREIRA, M. A.; RUDORFF, B. F. T.; FELICIO, J. C.; FREITAS, J. G.; TARGA, M. S. Variação espectral e eficiência do uso da radiação em ensaio com genótipos de trigo. Bragantia, Campinas, v. 64, n. 3, p.331-338, 2005.

PARAMESWARAN, K. V. M.; GRAHAM, R. D.; ASPINALL, D. Studies on the nitrogen and water relations of wheat II: effects of varying nitrogen and water supply on growth and grain yield. Irrigation Science, Berlim, v. 5, n. 2, p.105-121, apr. 1984.

RODRIGUES, O.; LHAMBY, J. C. B.; DIDONET, A. D.; MARCHESE, J. A.; SCIPIONI, C. Efeito da deficiência hídrica na produção de trigo. Pesquisa Agropecuária Brasileira, Brasília, v. 33, n. 6, p.839-846, 1998.

SCHEEREN, P. L. Informações sobre o trigo (Tritucum spp.). Passo Fundo: EMBRAPACNPT, 1986. 34p.

SILVA, A. R. A cultura do trigo irrigada nos cerrados do Brasil Central. Brasília: Embrapa-CPAC, 1978. 70p. (Circular técnica, 1)

SILVA, A. R.; LEITE, J. C. A cultura do trigo no cerrado com irrigação. In: REUNIÃO ANUAL CONJUNTA DE PESQUISA DE TRIGO, 7., Passo Fundo, 1975. Anais... Planaltina: Embrapa-CPAC, 1975. 4p. 